\title{
Generalized Fuzzy Time Series Forecasting Model Enhanced with Particle Swarm Optimization
}

\author{
Wangren Qiu ${ }^{1 *}$ Chunhua Zhang ${ }^{1}$ and Zhang Ping ${ }^{2}$ \\ ${ }^{1}$ Department of Information Engineering,Jingdezhen Ceramic Institute, Jingdezhen, \\ China, 333001 \\ ${ }^{2}$ Department of Economics,Hefei University of Technology, Heifei \\ 230009, Chinae \\ qiuone@163.com
}

\begin{abstract}
In the past two decades, many forecasting models based on the concepts of fuzzy time series have been proposed for dealing with various problem domains. In this paper, we present a novel model to forecast enrollments and the close prices of stock based on particle swarm optimization and generalized fuzzy logical relationships. After that some concepts of the generalized fuzzy logical relationships and an operation for combining the generalizedrelationships are introduced in the first part of this paper, we use particle swarm optimization to optimal represents for the given intervals in the universe of discourse to increase the forecasting accuracy. To test the effectiveness of the model, the proposed method is demonstrated on the procedure of forecasting enrollments as well as its experiment on forecasting the close price of Shanghai Stock Exchange Composite Index. Empirical analyses show that theproposed method gets a higher average forecasting accuracy rate than the existing methods.
\end{abstract}

Keywords:Fuzzy sets; Fuzzy forecasting; Generalized fuzzy logical relationship; particle swarm optimization

\section{Introduction}

Since fuzzy time series model was proposed by Song and Chissom[1-3] in 1993, there are many forecasting models have been developed to deal with the forecasting problems due to their capability of dealing with the uncertainty and vagueness inherent in the data collected. In the literatures, the experimental analysis existing in many areas, such as forecasting stock price [4-5], tourism demand[6], temperature[7], amount of export [8] and dry bulk shipping index [9], etc.

Most of the exiting methods mainly focused on partitioning the universe of discourse, constructing fuzzy relationships from the fuzzy set, forecasting and defuzzifing the forecasting output. A proper choice of the length of each interval can greatly improve the forecasting results. Huarng [10,11] presented some methods to obtain the proper lengths of the intervals for the fuzzy time series model. Chen proposed the automatic clustering technique to generate clustering-based intervals for some fuzzy time series forecasting models[12,13].Literatures[14,15]introduced an approach which using an optimization technique with a single-variable constraint to determine an optimal interval length. The particle swarm optimization algorithm and some other algorithms were used to search the appropriate intervals on the fuzzy time series[16-19] as well geneticalgorithms[20]. To improve the fuzzy time series model in constructing fuzzy relationships, forecasting and 
defuzzifing the forecasting output, Chen[21] modified the models by using simple algebraic operation in[1-3]. Chen introduced the Fibonacci sequence into the fuzzy time series, and testified the validity of the model[22]. Lee presented some high-order models based on two-factors and genetic simulated annealing techniques[23].

In fuzzy time series forecasting models, the midpoints, the represents of the given intervals, are critical for forecasting and defuzzifing the forecasting output. Although there are many models were proposed for deal with the lengths of intervals by combing with some modern algorithms [11-15] as well as particle swarm optimization[20,16,17]. The intervals are determined by the algorithms and the training data set, the midpoints of the improved intervals are the mean of endpoints of the related intervals. Unfortunately, the issue of how to determine proper represents for the given intervals has not been touched in previous studies. This paper proposes a new fuzzy time series model based on generalized fuzzy logical relationships and particle swarm optimization. The generalized fuzzy time series model was proposed to cope with the instability of the selection for fuzzy sets and improve the forecasted result[4]. At the same time, the particle swarm optimization is used to determine the propermidpoints of the given intervals. The proposed method is demonstrated on the enrollments of the University of Alabama. For the in-depth studies, we also applied the method to the close price of Shanghai Stock Exchange Composite Index (SSECI). The results are compared to the results of existing methods $[21,26]$.

\section{Preliminaries}

The frame work of Generalized fuzzy time series forecasting model can befound in reference [1-4]. Particle swarm optimization (PSO) is a population based stochastic optimization technique presented by Eberhart and Kennedy in $1995^{[24,25]}$, inspired by social behavior of bird flocking or fish schooling. That finding the global best solution by adjusting the trajectory of each individual towards its own best location and towards the best particle of the swarm is the main thought and shinning point of PSO which shares many similarities with evolutionary computation techniques such as Genetic Algorithms.

The particle swarm optimization concept consists of changing the velocity eachparticle toward its fitness value andglobal version of PSO. Acceleration is weighted by a random term, withseparate random numbers being generated for acceleration toward proper locations. The original process for implementing the local version of PSO is as follows:

(1)Initialize a population of particles with random positions and velocities on $d$ dimensions inthe problem space.

(2)For each particle, evaluate the desired optimization fitness function in $d$ variables.

(3)Compare particle's fitness evaluation with particle's pbest. If current value is better thanbest, then set pbest value equal to the current value, and the pbestlocation equal to the currentlocation in $d$-dimensional space.

(4)Compare fitness evaluation with the population's overall previous best. If current value is better thanlbest, then reset lbest to the current particle's array index and value.

(5)Change the velocity and position of the particle according to equations (1) and (2), respectively:

$$
\begin{array}{r}
V_{i d}=w * V_{i d}+C_{1} \operatorname{Rand}() *\left(P_{i d}-X_{i d}\right)+C_{2} \operatorname{Rand}() *\left(P_{l d}-X_{i d}\right) \\
X_{i d}=X_{i d}+V_{i d}
\end{array}
$$

where $w$ denotes the inertia weight factor; $X_{i d}$ is the current positionof the particle, $P_{i d}$ is the position of the particle that experiencesthe best fitness value; $P_{l d}$ is the location of the 
neighborhood best, the neighborhood size is defined as two in this paper; $C_{1}$ and $C_{2}$ are acceleration valueswhich represent the self-confidence coefficient and the social coefficient,respectively; $d$ denotes the dimension of the problem space; Rand( ) denotes a random function which can yield random value inthe range of $(0,1)$ and $V_{i d}$ denotes the velocity of the particle.

(6)Loop to step (2) until a criterion was met.

\section{Proposed Model}

In this subsection, an improved model of fuzzy time series basedon particle swarm optimization and generalized fuzzy logical relationship is proposed.Based on the classical procedure of FTS illuminated by Chen [21] and Lee [26], the presented model is given step-by-step as follows.

Step 1: Define the universe of discourse and intervals forrules extraction. Let $U=$ [starting,ending], according to the lengths of intervals, $U$ is partitioned into several intervals. For example, $U=\left\{u_{1}, u_{2}, \ldots, u_{n}\right\}$.In conventional models, $m_{i} i$ s the midpoint of $u_{i}$ whose corresponding fuzzy set is $A_{i}$. To enhance the representativeness, $m_{i}$ is replaced by the centroid point obtained with particle swarm optimization in the proposed method.

\section{Table 1.Fuzzified Enrollments and Fuzzy Logical Relationship}

\begin{tabular}{l}
\hline year actual value fuzzified fuzzy logical relationship \\
\hline $197113055 A_{1} ; A_{2} A_{1} \rightarrow A_{2} ; A_{2} \rightarrow A_{2}$ \\
$197213563 A_{2} ; A_{1} A_{2} \rightarrow A_{3} ; A_{3} \rightarrow A_{3}$ \\
$197313867 A_{3} ; A_{2} A_{3} \rightarrow A_{5} ; A_{2} \rightarrow A_{5}$ \\
$197414696 A_{5} ; A_{6} A_{5} \rightarrow A_{9} ; A_{6} \rightarrow A_{9}$ \\
$197515460 A_{9} ; A_{8} A_{9} \rightarrow A_{8} ; A_{8} \rightarrow A_{8}$ \\
$197615311 A_{8} ; A_{9} A_{8} \rightarrow A_{10} ; A_{9} \rightarrow A_{10}$ \\
$197715603 A_{10} ; A_{9} A_{10} \rightarrow A_{11} ; A_{9} \rightarrow A_{11}$ \\
$197815861 A_{11} ; A_{10} A_{11} \rightarrow A_{14} ; A_{10} \rightarrow A_{14}$ \\
$197916807 A_{14} ; A_{15} A_{14} \rightarrow A_{15} ; A_{15} \rightarrow A_{15}$ \\
$198016919 A_{15} ; A_{14} A_{15} \rightarrow A_{13} ; A_{14} \rightarrow A_{13}$ \\
$198116388 A_{13} ; A_{12} A_{13} \rightarrow A_{9} ; A_{12} \rightarrow A_{9}$ \\
$198215433 A_{9} ; A_{8} A_{9} \rightarrow A_{9} ; A_{8} \rightarrow A_{9}$ \\
$198315497 A_{9} ; A_{10} A_{9} \rightarrow A_{7} ; A_{10} \rightarrow A_{7}$ \\
$198415145 \quad A_{7} ; A_{6} A_{7} \rightarrow A_{7} ; A_{6} \rightarrow A_{7}$ \\
$198515163 A_{7} ; A_{8} A_{7} \rightarrow A_{11} ; A_{8} \rightarrow A_{11}$ \\
$198615984 A_{11} ; A_{12} A_{11} \rightarrow A_{15} ; A_{12} \rightarrow A_{15}$ \\
$198716859 A_{15} ; A_{14} A_{15} \rightarrow A_{17} ; A_{14} \rightarrow A_{17}$ \\
$198818150 A_{17} ; A_{18} A_{17} \rightarrow A_{19} ; A_{18} \rightarrow A_{19}$ \\
$198918970 A_{19} ; A_{20} A_{19} \rightarrow A_{21} ; A_{20} \rightarrow A_{21}$ \\
$199019328 A_{21} ; A_{20} A_{21} \rightarrow A_{15} ; A_{20} \rightarrow A_{21}$ \\
$199119337 A_{21} ; A_{20} A_{21} \rightarrow A_{19} ; A_{20} \rightarrow A_{19}$ \\
$199218876 A_{19} ; A_{18} A_{19} \rightarrow * ; A_{18} \rightarrow *$
\end{tabular}

Step 2: Define fuzzy sets based on the universe of discourse and intervalsand fuzzify the historical data. The fuzzy sets with respect to intervals given at Step 1 are defined with triangular fuzzy function and the membership degree of the value $x_{t}$ at time $t$ in $A_{i}$ be calculated by formula (3). 


$$
\mu_{A_{i}}(t)=\left\{\begin{array}{lc}
1 & \text { if } x_{t}>m_{n} \text { or } x_{t}<m_{1} \\
0 & \text { if } x_{t}<m_{i-1} \text { or } x_{t}>m_{i+1} \\
\frac{x_{t}-m_{i-1}}{m_{i}-m_{i-1}} & \text { if } m_{i-1} \leq x_{t} \leq m_{i} \\
\frac{m_{i+1}-x_{t}}{m_{i+1}-m_{i}} & \text { if } m_{i} \leq x_{t} \leq m_{i+1}
\end{array}\right.
$$

where $x_{t}$ is the observed value at time $t, m_{i}$ is the centroid point of interval $u_{i}$ which corresponding to main interval of fuzzy set $A_{i}$.

Step 3: Establish the fuzzy logical relationships based on the hierarchies of principal fuzzy logical relationship. Given the sample data set and the definition of fuzzy sets, all fuzzy logical relationships between two consecutive data can be established. To forecast the time series, the fuzzy logical relationship matrix must be created at this step based on the fuzzy logical relationships.

Step 4: Forecasting with the fuzzy relation matrices. Let the membership values of $F(t)$ with respect to the given fuzzy sets are $\left(\mu_{1}(t), \mu_{2}(t), \ldots, \mu_{n}(t)\right)$ and $\mu_{t_{l}}(t)$ is the lth maximum membership degree, respectively. Wehave the intersection of $N$ fuzzy logical relationship groups, and then theforecasting value $F(t+1)$ is conducted by formula (4).

$$
F(t+1)=\Lambda_{\mathrm{N}}\left(R^{(1)}\left(i_{1},:\right), \cdots, R^{(l)}\left(i_{l},:\right), \cdots R^{(N)}\left(i_{N},:\right)\right)^{\circ}\left(m_{1}, m_{2}, \cdots, m_{n}\right)^{\mathrm{T}}
$$

where $^{\circ}$ is a composition operation for forecasting with two principles:(1) If the sum of $\wedge_{\mathrm{N}}\left(R^{(1)}\left(i_{1},:\right), \cdots, R^{(l)}\left(i_{l},:\right), \cdots R^{(N)}\left(i_{N},:\right)\right)$ equals to 0 , then the forecastis $m_{t_{1}}$ which is the midpoint of the intervalcorresponding to $A_{t_{1}}^{(1)} ;(2)$ Otherwise, the forecasted value is the weighted sum of $m_{1}, m_{2}, \cdots, m_{n}$.

Since most of conventional models have been presented for forecasting the historical enrollments of the University of Alabama, in this section, we present stepwise procedures of the proposed method for forecasting the time series data with $N=2$ and $p=1$. The well-known data, historical enrollments of the University of Alabama from 1971 to 1992 , are shown in Table 1.

Step 1: Partition the universe of discourse $U$ with the automatic clustering technique.The intervals obtained by the algorithm can belisted as $u_{1}=[13055,13354)$, $u_{2}=[13354,13862), \ldots, \quad u_{21}=[19328,19337)$. Their midpoints are $m_{1}=13205$, $m_{2}=13608, \ldots, m_{21}=19332$ respectively.

Table 2.Fuzzy Logical Relationships Groups when $N=2$

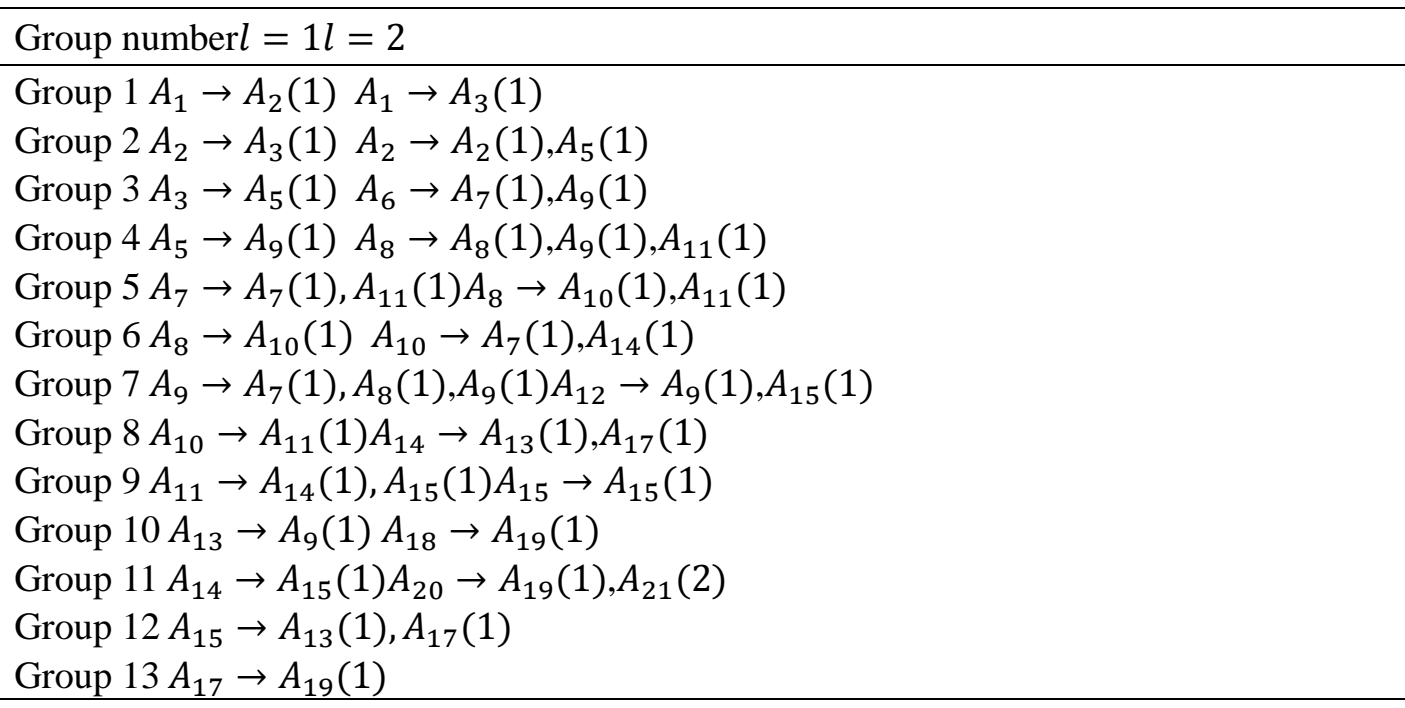


Group $14 A_{18} \rightarrow A_{21}(1)$

Group $15 A_{21} \rightarrow A_{19}(1), A_{21}(1)$

Step 2: Fuzzify each datum in the training data set into afuzzy set defined in Step 1.With triangular-shaped membership function defined as formula(4), the fuzzy sets and all observations are definedas the second column of Table 1 by formula(4). For example, the datum is 13055 in 1971, the membership degrees then are $(1.0000,0.3147,0, \ldots, 0, \ldots, 0)$. With $\mathrm{N}=2$, the maximum value is 1 which is the membership degree of $A_{1}$ and the second maximum value is 0.3147 which is the membership degree of $A_{2}$, therefore, the datum of 1971 be fuzzified as $A_{1}$ and $A_{2}$. For $N=2$, all of the training data are fuzzified with this method and listed in the third and fourth column of Table 1.

Step 3: Construct the fuzzy logical relationships based on thefuzzified training data obtained in Step 2 and create the fuzzy relation matrix on the basis of fuzzy logical relationship groups.

From Table 2, the obtained fuzzy logical relationships are listed in the fifth and sixth column of the Table. The fuzzy logical relationships are grouped and listed in Table 2. The weights of the fuzzy logical relationship are counted by the recurrent fuzzy relationships with using the method of Lee's [25].

Table 3. Forecasts of Enrollment with $N=2$

\begin{tabular}{|c|}
\hline$p=1 p=2 p=3 p=4 p=5 p=6$ \\
\hline 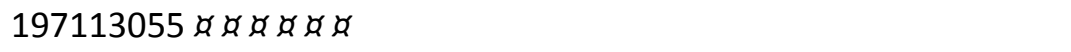 \\
\hline 197213563136081348113608135451360813566 \\
\hline 197313867140141393813913139001389313887 \\
\hline 197414696146961484614696146211469614646 \\
\hline 197515460154571538415457154941545715481 \\
\hline 197615311153471540215217152931529615299 \\
\hline 197715603157321566815646156351562915625 \\
\hline 197815861159231589215882158761587315871 \\
\hline 197916807167471683516826168211681816816 \\
\hline 198016919168631689116900169051690816910 \\
\hline 198116388172691623816388163131638816338 \\
\hline 198215433154571538415457154211545715433 \\
\hline 198315497153471540215457154941551515481 \\
\hline 198415145151541515015217151471514715147 \\
\hline 1985151631515415159151601516115161 \\
\hline 198615984159231601016001159971599415993 \\
\hline 198716859168631683516863168491686316854 \\
\hline 198818150172691830018150180751815018100 \\
\hline 198918970189231894718954189581896118962 \\
\hline 199019328193331933519330193291932919329 \\
\hline 199119337191281911719336193361933619336 \\
\hline 199218876191281911718892188881888518884 \\
\hline
\end{tabular}

Step 4: Calculate the forecasts. To carry out the calculationsby the proposed principles, we 
illustrate the forecasting process of enrollment of 1972 and 1991 as follows.With the definition of membership function (4), the membership degrees of enrollment of 1972 is $(0.5573,0.9441,0.4427,0, \ldots, 0)$. The two maximum membership attributesare of the second and the first fuzzy sets. Then, the second row of $R^{(1)}$ is $(0,0,1,0, \ldots, 0)$ andthe first row of $R^{(2)}$ is $(0,0,1,0, \ldots, 0, \ldots, 0)$. We have $\Lambda_{\mathrm{N}}\left(R_{1972_{1}}^{(1)}, R_{1972_{2}}^{(1)}\right)=(0,0,1, \ldots, 0)$. According to the second principle $F(1973)$ then is $m_{3}$, i.e. 14014 , which is themiddle point of $A_{3}$. Table 3 shows the actual enrollments and the forecasted enrollments obtained by using the proposed method with $p=1,2, \ldots, 6$.

Table 4. A Comparison of MSE of the Proposed Method with the Existing Methods

\begin{tabular}{|c|}
\hline $\begin{array}{r}\text { YearActualvalue }[21][10][18][19][12]_{p=1}[12]_{p=5} p=1 p=1 \\
N=1 N=5\end{array}$ \\
\hline 197113055 \\
\hline 1972135631400014000142301424213608136081360813608 \\
\hline 1973138671400014000142301424214014138921401413893 \\
\hline 1974146961400014000142301424214696146961469614696 \\
\hline 1975154601550015500155411547415467154671545715457 \\
\hline 1976153111600015000155411547415455153141534715296 \\
\hline 1977156031600016000155411547415455156281573215629 \\
\hline 1978158611600016000161961547415923158731592315873 \\
\hline 1979168071600016000161961614716863168181674716818 \\
\hline 1980169191683317500161961698817768170121686316908 \\
\hline 1981163881683316000175071698816388163881638816388 \\
\hline 1982154331683316000161961614715467154121545715457 \\
\hline 1983154971600016000155411547415455155211545715515 \\
\hline 1984151451600015500155411547415455151461515415147 \\
\hline 1985151631600016000155411547415247151791515415161 \\
\hline 1986159841600016000155411547416037159941592315994 \\
\hline 1987168591600016000161961614716863168631686316863 \\
\hline 1988181501683317500175071698817768181501815018150 \\
\hline 1989189701900019000188721914419149190051892318961 \\
\hline 1990193281900019000188721914419333193281933319329 \\
\hline 1991193371900019500188721914419128193361912819336 \\
\hline 1992188761900019000188721914419128188851892318885 \\
\hline MSE 407507229516261162228920565807125072248 \\
\hline RMSE638 479511478238277116 \\
\hline MAE 499398429386143164711 \\
\hline MAPE 0.031100 .024830 .026600 .023960 .008570 .001030 .002900 .00072 \\
\hline
\end{tabular}

\section{Empirical Analysis}

For that it is simple and easy to display the process of the forecasting models, enrollment data of University of Alabama is forecasted in this paper and widely served the experiment of fuzzy timeseries studies. Since time-series models have been used to makepredictions in the areas of stock price forecasting for many years,the daily Shanghai Stock Exchange Composite Index closing pricescovering the period from 1997 to 2006 are adopted as thetraining dataset for further analysis. 
In statistics, the Mean Squared Error (MSE), the Root Mean Squared Error (RMSE), Mean AbsoluteError (MAE) and Mean Absolute Percentage Error (MAPE) are sometypical criteria for quantifying the difference between values implied by anestimator and the actual values of the quantity being estimated. MSEis a risk function for measures the average of the squares of thedifference. For an unbiased estimator, the MSE is the variance, andthe RMSE is the square root of the variance known as the standarderror. Furthermore, RMSE has over MSE because its scale is the sameas the forecasts. As an average of the absolute percent errors, MAPE is another criterion for the comparisons of forecasting results in the paper.

Table 5. Comparison of Forecasting Enrollment

\begin{tabular}{|c|c|}
\hline & $N p=1 p=2 p=3 p=4 p=5 p=6$ \\
\hline 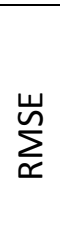 & $\begin{array}{l}\mathrm{N}=1317.55213 .9658 .4741 .2032 .3433 .38 \\
\mathrm{~N}=2287.92102 .2233 .8932 .4215 .7521 .51 \\
\mathrm{~N}=3282.8287 .7933 .8932 .4215 .7521 .51 \\
\mathrm{~N}=471.2287 .7933 .8932 .4215 .7521 .51 \\
\mathrm{~N}=571.2287 .7933 .8932 .4215 .7521 .51\end{array}$ \\
\hline$\underset{\Sigma}{\stackrel{u}{\Sigma}}$ & 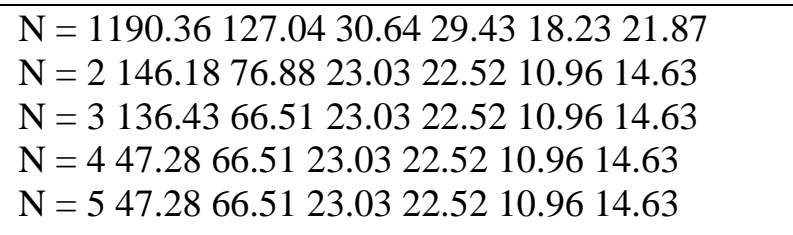 \\
\hline$\frac{\omega}{\underline{a}}$ & 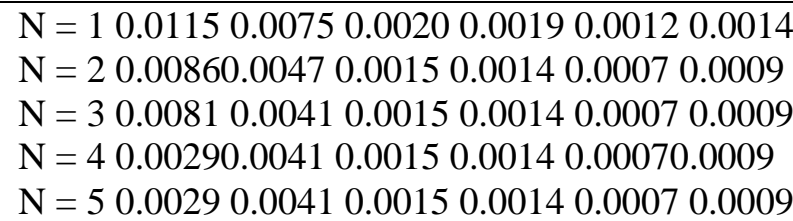 \\
\hline
\end{tabular}

In Table 4, this study makes a comparison of the forecasted results ofthe proposed method with Chen's method[3], Cheng et al.'smethods[8,10], Huarng's method[15], Chen'smethod[6] with $\mathrm{p}=1$ and $p=5$.From the Table 4, we can see that the proposed method has a forecasting error than Chen's method, Cheng et al.'smethods on the basis of the four criteria of evaluation. With the same $p$, the proposed method also has a smaller forecasting error than that of Chen's. In other words, the proposedmethod gets a higher average forecasting accuracy rate than these counterparts.

Table 6. Comparison of Forecasting SSECI of 1997

\begin{tabular}{l|l}
\hline & \multicolumn{1}{|c}{$N p=1 p=2 p=3 p=4 p=5 p=6$} \\
\hline & $\mathrm{N}=111.579 .188 .344 .203 .403 .50$ \\
& $\mathrm{~N}=29.604 .763 .392 .332 .701 .83$ \\
$\sum_{\propto}$ & $\mathrm{N}=39.474 .763 .392 .332 .701 .83$ \\
& $\mathrm{~N}=48.834 .683 .362 .222 .641 .74$ \\
& $\mathrm{~N}=58.834 .683 .362 .222 .641 .74$ \\
\hline
\end{tabular}




\begin{tabular}{|c|c|}
\hline$\underset{\Sigma}{\stackrel{u}{\Sigma}}$ & 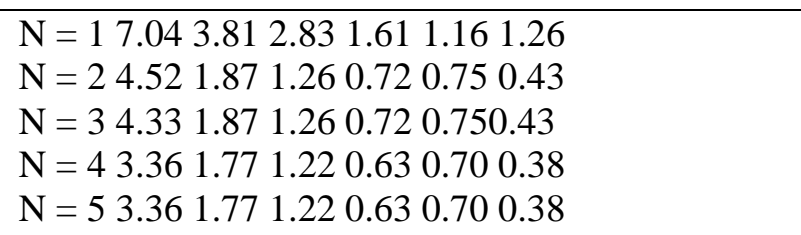 \\
\hline$\frac{\omega}{\grave{a}}$ & 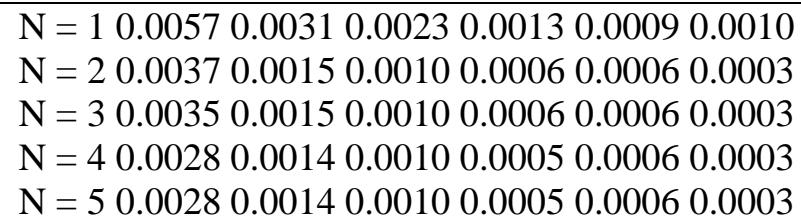 \\
\hline
\end{tabular}

To further investigate the effect of the hierarchies of fuzzy logical relationships on the forecasting results, we apply the proposed method to handle forecasting enrollments with different $p$ and $N$.Table 5 shows the forecasted RMSE, MAE and MAPE obtained by using the proposed method with $p=1,2, \ldots, 6$ and $N=1,2, \ldots, 5$.

From the table, we can arrive at two conclusions: firstly, the forecasting errors will reduce while $N$ is increasing for a given $p$; secondly, the proposed model can obtain higher forecast forecasting accuracy rate with an increasing $p$ for a given $N$.

Table 7. Comparison of Forecasting SSECI of 1998

\begin{tabular}{|c|c|}
\hline & $N p=1 p=2 p=3 p=4 p=5 p=6$ \\
\hline 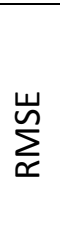 & $\begin{array}{l}\mathrm{N}=1 \quad 11.579 .18 \quad 8.344 .203 .403 .50 \\
\mathrm{~N}=29.604 .763 .392 .332 .701 .83 \\
\mathrm{~N}=39.474 .763 .392 .332 .701 .83 \\
\mathrm{~N}=48.834 .683 .362 .222 .641 .74 \\
\mathrm{~N}=58.834 .683 .362 .222 .641 .74\end{array}$ \\
\hline$\underset{\Sigma}{\Psi}$ & $\begin{array}{l}\mathrm{N}=17.043 .812 .831 .611 .161 .26 \\
\mathrm{~N}=24.521 .871 .260 .720 .750 .43 \\
\mathrm{~N}=34.331 .871 .260 .720 .750 .43 \\
\mathrm{~N}=43.361 .771 .220 .630 .700 .38 \\
\mathrm{~N}=53.361 .771 .220 .630 .700 .38\end{array}$ \\
\hline 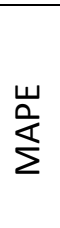 & 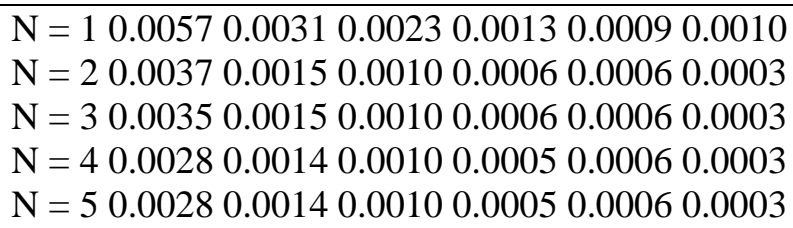 \\
\hline
\end{tabular}

Table 8. Comparison of Mean forecasting SSECI

\begin{tabular}{l|l}
\hline & \multicolumn{1}{|c}{$N p=1 p=2 p=3 p=4 p=5 p=6$} \\
\hline & $\mathrm{N}=113.488 .196 .155 .304 .723 .91$ \\
& $\mathrm{~N}=210.935 .654 .283 .743 .302 .86$ \\
$\sum_{\propto}$ & $\mathrm{N}=310.845 .644 .283 .743 .302 .86$ \\
& $\mathrm{~N}=48.174 .974 .033 .162 .932 .59$ \\
& $\mathrm{~N}=58.174 .974 .033 .162 .932 .59$ \\
\hline
\end{tabular}




\begin{tabular}{|c|c|}
\hline$\underset{\Sigma}{\stackrel{\amalg}{\Sigma}}$ & 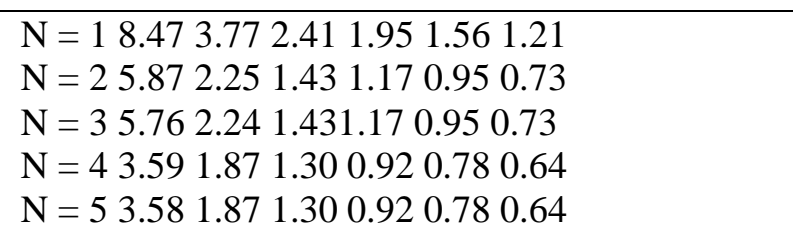 \\
\hline$\stackrel{\omega}{\frac{\omega}{\alpha}}$ & 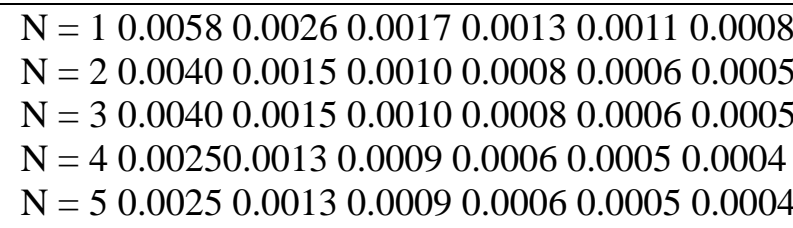 \\
\hline
\end{tabular}

To test the universality of the conclusions, the proposed model has also been used to forecast the close price of SSECI covering the ten years period from 1997 to 2006. Table 6 and 7 show the forecasted RMSE, MAE and MAPE of SSECI in 1997 and 1998. Table 8 illustrates the mean forecasted RMSE, MAE and MAPE of SSECI from 1997 to 2006. From these three tables, we can easy to accept the above conclusions as true.

Some example of actual values and forecasts of1997 are depicted in Figures 1 and 2. Figure 1 tells us that the performance of the proposed model is improving a lot with the increasing $\mathrm{N}$ in the same number of intervals. Figure 2 illustrates that the proposed model obtains the better forecasts when the number of intervals are increasing. All of these conclusions have also been depicted by Table 6,7 and 8 with the three criteria.

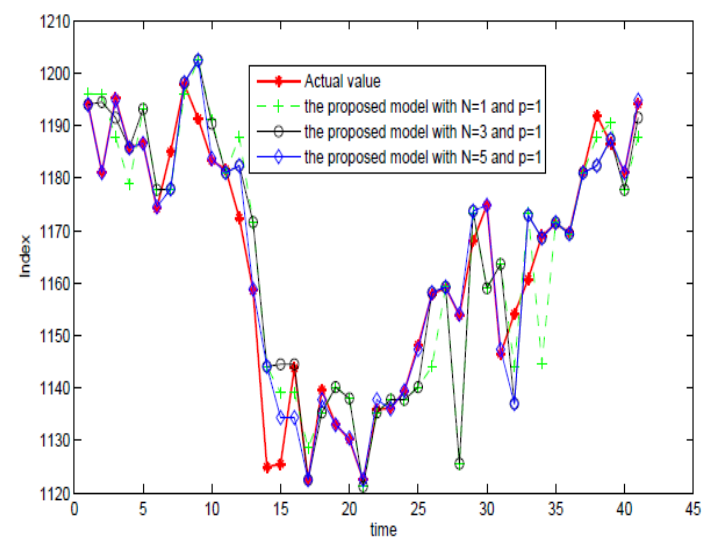

Figure 1.Forecasts of 1997 with Different N

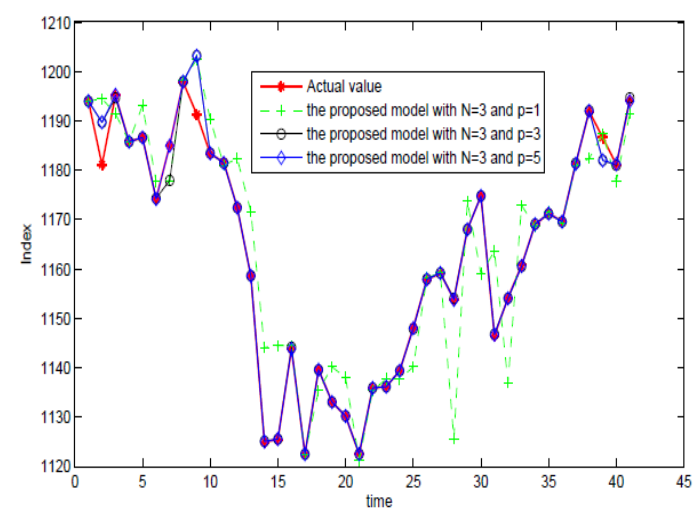

Figure 2. Forecasts of 1997 with Different $p$ 


\section{Conclusion}

It is critical to determine the proper represents of the given intervals besides the determination of effective lengths of intervals for forecasting in fuzzy time series. The objective of this study is to provide a method for proper represents of intervals to improve forecasting with generalized fuzzy time series model. In the proposed method, the proper represents of intervals are obtained by using local version of standard particle swarm optimization to find the minimum value of the forecasted errors. The new proposed method is applied to well-known enrollment data as well as the close price of SSECI. Obtained results are compared with other methods proposed in the literature.

To sum up, the empirical analyses for different applications show that the proper represents of intervals outperformed most of the other midpoints of intervals. Hence, except for the superiority of generalized fuzzy time series model, this study also suggests that finding an optimal represents for given intervals can be applied to improve fuzzy time series forecasting.

\section{Acknowledgements}

This work was partially supported by the National Nature Science Foundation of China (NSFC 61261027), Natural ScienceFoundation of Jiangxi Province, China (20142BAB207013), and Foundation of Jiangxi Province Educational Committee (GJJ14642,GJJ14651).

\section{References}

[1] Q. Song and B. S. Chissom, "Fuzzy time series and its models", Fuzzy Sets System, vol. 54, no. 3, (1993), pp. 269-277.

[2] Q. Song and B. S. Chissom, "Forecasting enrollments with fuzzy time series", Part I. Fuzzy Sets System, vol. 54, no. 1, no. 1-9, (1993).

[3] Q. Song and B. S. Chissom, "Forecasting enrollments with fuzzy time series", Part II, Fuzzy Sets System, vol. 62 , no. 1, (1994), pp. 1-8.

[4] W. R.Qiu, X. D. Liu and L. D. Wang, "Forecasting in time series based on generalized fuzzy logical relationship", ICIC Express Letters, vol. 4, no. 5, (2010), pp. 1431-1438.

[5] W. R. Qiu, X. D. Liu and H. L. Li, "A Generalized Method for Forecasting Based on Fuzzy Time Series", Expert Systems with Applications, vol. 38, no. 8, (2011), pp. 10446-10453.

[6] H. L.Chou, J. S. Chen and C. H. Cheng, "Forecasting Tourism Demand Based on Improved Fuzzy Time Series Model”, Intelligent Information and Database Systems Lecture Notes in Computer Science, (2010), pp. 5990,399-407.

[7] L. W. Lee, L. H. Wang and S. M. Chen, "Temperatureprediction and TAIFEX forecasting based on fuzzy logicalrelationships and genetic algorithms", Expert Systems withApplications, vol. 33, (2007), pp. 539-550.

[8] H. L. Wong, Y. H. Tu and C.C. Wang, "Application of fuzzy time series models for forecasting the amount of Taiwan export”, Expert Systems with Applications, vol. 37, (2010), pp. 1465-1470.

[9] D. Okan, "A fuzzy integrated logical forecasting model for dry bulk shipping index forecasting: An improved fuzzy time series approach", Computers and Mathematics with Applications, vol. 37, (2010), pp. 5372-5380.

[10] K. H. Huarng, "Heuristic models of fuzzy time series for forecasting”, Fuzzy Sets and Systems, vol. 123, no. 3, (2001), pp. 369-386.

[11] K. H. Huarng, "Ratio-based lengths of intervals to improve fuzzy time series forecasting", IEEE Transactions on Systems, Man, and Cybernetics-Part B: Cybernetics, vol. 36, no. 2, (2006), pp. 328-340.

[12] S. M. Chen, N. Y. Wang and J. S. Pan, "Forecasting enrollments using automatic clustering techniques and fuzzy logical relationships", Expert Systems with Applications, vol. 36, (2009), pp. 11070-11076.

[13] N. Y. Wang and S. M. Chen, "Temperature prediction and TAIFEX forecasting based on automatic clustering techniques and two-factors high-order fuzzy time series", Expert Systems with Applications, vol. 36, (2009), pp. 2143-2154.

[14] E. Egrioglu, C. H. Aladag, U. Yolcu, V. R. Rslu and M. A. Basaran, "Finding an optimal interval length in 
high order fuzzy time series”, Expert Systems with Applications, vol. 37, (2010), pp. 5052-5055.

[15 Y. U. Fuk, E. Erol, R. U. Vedide, A. B. Murat and H. A. Cagdas, "A new approach for determining the length of intervals for fuzzy time series", Itextit\{Applied Soft Computing\}, vol. 9, no. 2, (2009), pp. 647-651.

[16] I. H. Kuo, S. J. Horng, Y. H. Chen, R. S. Run, T. W. Kao, R. J. Chen, J. L. Lai and T. L. Lin, "Forecasting TAIFEX based on fuzzy time series and particle swarm optimization", Expert Systems with Applications, vol. 37, (2010), pp. 1494-1502.

[17] S. T. Li, Y. C. Cheng and S. Y. Lin, "A FCM-based deterministic forecasting model for fuzzy time series", Computers and Mathematics with Applications, vol. 56, (2008), pp. 3052-3063.

[18] C. H. Cheng, J. R. Chang and C. A. Yeh, "Entropy-based and trapezoid fuzzification-based fuzzy time series approaches for forecasting IT project cost", Technological Forecasting and Social Change, vol. 73, no. 5, (2006), pp. 524-543.

[19] C. H. Cheng, G. W. Cheng and J. W. Wang, "Multi-attribute fuzzy time seriesmethod based on fuzzy clustering”, Expert Systems with Application, vol. 34, no. 2, (2008), pp. 1235-1242.

[20] S. M. Chen and N. Y. Chung, "Forecasting enrollments using high-order fuzzy time series and genetic algorithms", International Journal of Intelligent Systems, vol. 21, no. 5, (2006),pp. 484-501.

[21] S. M. Chen, "Forecasting enrollments based on fuzzy time series", Fuzzy Sets and Systems, vol. 81, (1996), pp. 311-319.

[22] T. L. Chen, C. H. Cheng and H. J. Teoh, "Fuzzy time-series based on Fibonacci sequence for stock price forecasting”, Physica A. Elsevier, London, vol. 380, (2007), pp. 377-390.

[23] L. W. Lee, L. H. Wang, M. S. Chen and Y. H. Leu, "Handling Forecasting Problems Based onTwo-Factors High-Order Fuzzy Time Series", Ieee Transactions on fuzzy systems, vol. 14, no. 3, (2006), pp. 468-477.

[24] R. C. Eberhart and Y. H. Shi, "Particle swarm optimization: developments, applications and resources", Evolutionary Computation, Proceedings of the Congress, (2001).

[25] J. Kennedy and R. Eberhart, "Particle swarm optimization", Proceedings of IEEE International Conference on Neural Networks, IEEE Press, (1995); Piscataway, NJ, USA.

[26] M. H. Lee, E. Riswan and I. Zuhaimy, "Modified Weighted for Enrollment Forecasting Based on Fuzzy Time SeriesC", MATEMATIKA, vol. 25, no. 1, (2009), pp. 67-78. 
International Journal of $u-$ and e- Service, Science and Technology Vol.8, No.5 (2015) 